\title{
Purification of chicken carbonic anhydrase isozyme-III (CA-III) and its measurement in White Leghorn chickens
}

\author{
Toshiho Nishita ${ }^{1 *}$, Yuichiro Tomita ${ }^{2}$, Daisuke Yorifuji ${ }^{1}$, Kensuke Orito ${ }^{3}$, Hideharu Ochiai ${ }^{4}$ and Kazuyosi Arishima ${ }^{5}$
}

\begin{abstract}
Background: The developmental profile of chicken carbonic anhydrase-III (CA-III) blood levels has not been previously determined or reported. We isolated CA-III from chicken muscle and investigated age-related changes in the levels of CA-III in blood.

Methods: CA-III was purified from chicken muscle. The levels of CA-III in plasma and erythrocytes from 278 female chickens (aged 1-93 weeks) and 68 male chickens (aged 3-59 weeks) were determined by ELISA.

Results: The mean level of CA-III in female chicken erythrocytes (1 week old) was $4.6 \mu \mathrm{g} / \mathrm{g} \mathrm{of} \mathrm{Hb}$, and the CA-III level did not change until 16 weeks of age. The level then increased until 63 weeks of age $(11.8 \mu \mathrm{g} / \mathrm{g} \mathrm{of} \mathrm{Hb})$, decreased to $4.7 \mu \mathrm{g} / \mathrm{g}$ of $\mathrm{Hb}$ at 73 weeks of age, and increased again until 93 weeks of age (8.6 $\mu \mathrm{g} / \mathrm{g}$ of Hb). The mean level of CA-III in erythrocytes from male chickens (3 weeks old) was $2.4 \mu \mathrm{g} / \mathrm{g}$ of Hb , and this level remained steady until 59 weeks of age. The mean plasma level of CA-III in 1-week-old female chickens was $60 \mathrm{ng} / \mathrm{mL}$, and this level was increased at 3 weeks of age $(141 \mathrm{ng} / \mathrm{mL})$ and then remained steady until 80 weeks of age (122 ng/ $\mathrm{mL}$ ). The mean plasma level of CA-III in 3-week-old male chickens was $58 \mathrm{ng} / \mathrm{mL}$, and this level remained steady until 59 weeks of age.

Conclusion: We observed both developmental changes and sex differences in CA-III concentrations in White Leghorn (WL) chicken erythrocytes and plasma. Simple linear regression analysis showed a significant association between the erythrocyte CA-III level and egg-laying rate in WL-chickens $16-63$ weeks of age $(p<0.01)$.
\end{abstract}

\section{Background}

Carbonic anhydrase (CA; EC 4.2.1.1), a well-characterized enzyme, catalyzes the reversible hydration of $\mathrm{CO}_{2}$ to form $\mathrm{HCO}_{3}{ }^{-}$and protons according to the following reaction: $\mathrm{CO}_{2}+\mathrm{H}_{2} \mathrm{O} \leftrightarrow \mathrm{HCO}_{3}^{-}+\mathrm{H}^{+}$. The enzyme can (reversibly) convert carbon dioxide into bicarbonate and hydrogen ions without the formation of carbonic acid. The first reaction is catalyzed by carbonic anhydrase and the second occurs instantaneously. CA plays important roles in gas transport, acid/base regulation, bone resorption and calcification, ion transport, and various secretory functions in several tissues [1]. There are at least 11 active CA isozymes (cytosolic CA-I, CA-II, CA-III, and CA-VII, mitochondrial CA- $V_{A}$ and $V_{B}$, secretory CA-VI,

\footnotetext{
* Correspondence: nishida@azabu-u.ac.jp

'Laboratories of Veterinary Physiology 1, School of Veterinary Medicine,

Azabu, University,1-17-71 Fuchinobe, Sagamihara, Kanagawa Japan 252-5201

Full list of author information is available at the end of the article
}

and membrane-associated CA-IV, IX, XII, and XIV), as well as 3 inactive CA-related proteins (CA-VIII, X, and $\mathrm{XI})[2,3]$. Each isozyme has a unique molecular structure. CA-III has been demonstrated in human [4], horse [5], cow [6], sheep [7], rabbit [8], dog [9], pig [10], and chicken [7] skeletal muscle. Among the isozymes, CA-III has the lowest turnover rate and greatest resistance to inhibition by sulphonamides. CA-III may facilitate the transport of $\mathrm{CO}_{2}$ in skeletal muscle by catalyzing the reversible hydration of $\mathrm{CO}_{2}$ to the more soluble $\mathrm{HCO}_{3}{ }^{-}$. Raisanen et al. [11] suggested that CA-III is an oxyradical scavenger and thus protects cells from oxidative damage. CA-III is relatively specific to skeletal muscle $[4,12]$ and could thus be useful as a diagnostic marker for muscle disease $[13,14]$.

We found no previous reports on CA-III blood concentrations in healthy chickens among the studies on chicken CA-III published thus far. The present study

\section{C) Biomed Central}


aimed to establish simple methods for the purification of chicken CA-III and develop an enzyme-linked immunoassay (ELISA) system to measure CA-III levels in erythrocytes and plasma from normal WL chickens.

\section{Methods}

\section{Purification of chicken CA-III}

The leg muscles were excised from several female white Leghorn (WL) chickens killed by an overdose of Nembutal. WL-chickens. Sample of muscle, weighing $200 \mathrm{~g}$, was homogenized at $4^{\circ} \mathrm{C}$ in a blender containing $400 \mathrm{~mL}$ of $0.01 M$ Tris- $\mathrm{HCl}$ buffer ( $\mathrm{pH}$ 8.0). All experiments were performed according to the guidelines of The Laboratory Animal Care Committee of Azabu University and in compliance with The Japanese Animal Welfare Guide. The homogenate was centrifuged at $27,000 \times$ g for $30 \mathrm{~min}$ at $4^{\circ}$ C. Iodoacetamide was then added to a final concentration of $0.01 \mathrm{M}$, the $\mathrm{pH}$ of the solution was adjusted to 8.0, and the mixture was incubated for 30 min under non-denaturing conditions. The sample was dialyzed against $0.01 \mathrm{M} \mathrm{2-}$ ( $N$-morpholino) ethanesulfonic acid (MES) buffer ( $\mathrm{pH}$ 6.0) (Dojindo Laboratories, Kumamoto, Japan) at $4^{\circ} \mathrm{C}$. The dialyzed sample was centrifuged at 27,000 $\times$ g for $30 \mathrm{~min}$ at $4^{\circ} \mathrm{C}$, and the supernatant was applied to a carboxymethyl (CM)-cellulose column (CM 52, Whatman International Ltd., Maidstone, UK) $(3.4 \times 30 \mathrm{~cm})$ equilibrated with 0.01 $M$ MES buffer ( $\mathrm{pH}$ 6.0). After extensive washing, adsorbed proteins were eluted with a linear gradient of 0-0.15 M $\mathrm{NaCl}$ in 0.01 $M$ MES buffer ( $\mathrm{pH} \mathrm{6.0)}$ ) at a flow rate of $20 \mathrm{~mL} / \mathrm{h}$ using a peristaltic pump. The eluate was collected in $6 \mathrm{~mL}$ fractions. The optical densities at $280 \mathrm{~nm}$ of all fractions were recorded. Fractions with enzymatic activity were collected and pooled. Pooled samples were precipitated with saturated ammonium sulfate, and the precipitate was collected by centrifugation. Precipitates were then dissolved in a small amount of distilled water. Samples were next passed at a flow rate of $15 \mathrm{~mL} / \mathrm{h}$ over a Sephacryl S-200 HR (Sephacryl HR, Pharmacia Biotech, Uppsala, Sweden) column equilibrated with $0.05 \mathrm{M}$ Tris$\mathrm{HCl}$ (pH 8.0) containing $0.5 \mathrm{M} \mathrm{NaCl}$. The major peak had $\mathrm{CA}$ activity and was collected and dialyzed against water. Samples were further purified by the column electrofocusing method of Svenson [15] using an electrofocusing column and Ampholine ( $\mathrm{pH}$ 8.0-10.5, Pharmacia Biotech, Uppsala, Sweden). The fraction containing CA activity was dialyzed against $0.01 M$ Tris- $\mathrm{HCl}$ buffer ( $\mathrm{pH} 7.5$ ). Purified chicken CA-III was stored at $-80^{\circ} \mathrm{C}$ until use.

\section{Characterization of purified chicken CA-III Partial amino acid sequencing of chicken CA-III}

Purified chicken CA-III was cleaved with endoproteinase Glu-C (Sigma-Aldrich Corp., St. Louis, MO, USA), in the presence of sodium dodecyl sulfate (SDS), according to the Cleveland method [16]. Digested chicken CA-III was separated by sodium dodecyl sulfate polyacrylamide gel electrophoresis (SDS-PAGE). One of the main peptide bands was sequenced using a Procise 491 protein sequencer (Applied Biosystems, Foster City, CA, USA) to obtain a partial amino acid sequence.

\section{Electrophoretic procedures and western blotting}

SDS-PAGE was performed using 12.5\% PhastGel Homogeneous gels and the PhastSystem (Pharmacia Biotech, Uppsala, Sweden). Thin-layer isoelectric focusing (IEF) was performed with PhastGel IEF media and the PhastSystem. A low molecular weight calibration kit and isoelectric point calibration kit (Pharmacia Biotech) were used to determine the molecular weight and isoelectric point of the protein. After electrophoresis, the gel was stained with Coomassie Brilliant Blue.

Western blotting was performed as previously described [17]. Briefly, adequate volumes of hemolysate and purified chicken CA-III were separated on 12.5\% PhastGel Homogeneous gels and transferred to Immobilon PVDF transfer membrane (Millipore Corp, Bedford, Mass, USA) using a commercially available transfer system. The buffer for electrophoretic transfer contained $25 \mathrm{~m} M$ Tris ( $\mathrm{pH} 8.3$ ), $192 \mathrm{~m} M$ glycine, $0.1 \%$ SDS, and $15 \%$ methanol. CA-III was detected using rabbit anti-chicken CA-III antiserum produced in the present study. This antiserum was diluted 1:2000 in $5 \mathrm{~mL}$ of $50 \mathrm{mM}$ Tris- $\mathrm{HCl}$ ( $\mathrm{pH} \mathrm{7.5)} \mathrm{containing}$ $0.3 \%$ BSA, $0.9 \% \mathrm{NaCl}, 0.01 \%$ thimerosal, and $10 \mathrm{mM}$ EDTA (buffer A). After incubation with the primary antibody, membranes were washed in $0.15 \mathrm{M}$ phosphatebuffered saline solution (PBS) containing 0.05\% Tween (PBS-Tween) and incubated with peroxidase-conjugated goat anti-rabbit immunoglobulin G (IgG) antiserum (Kirkegaard \& Perry Laboratories Inc., Gaithersburg, CA, USA) diluted 1:4,000 in $5 \mathrm{~mL}$ of buffer A. Membranes were washed again with PBS-Tween and developed by incubation with $0.02 \%$ hydrogen peroxide and $0.2 \mathrm{mM}$ diaminobentidine-tetrahydrochloride in $0.05 \mathrm{M}$ Tris- $\mathrm{HCl}$ (pH 7.6) for approximately $5 \mathrm{~min}$.

\section{Enzyme analysis}

The enzymatic activity of CA was measured using the method of Wilbur and Anderson [18]. Assays were performed at $4^{\circ} \mathrm{C}$, and specific activity (U) was determined according to the formula:

$$
\mathrm{U}=10 \times(\mathrm{Tb} / \mathrm{Te}-1) / \mathrm{mg} \text { of protein }
$$

Where $\mathrm{Tb}$ is the time required for the reaction (i.e., $\mathrm{pH}$ change from 8.5 to 6.5 ) in the absence of enzyme and $\mathrm{Te}$ is the time required for the same reaction in the presence of enzyme. The reaction time was measured with a stopwatch. 


\section{Immunochemistry}

Antibodies against purified chicken CA-III were produced in rabbits. Each rabbit was immunized initially with $1 \mathrm{mg}$ of purified CA-III emulsified with an equal volume of Freund's complete adjuvant. This was followed by a booster injection of an equivalent amount of enzyme once a week for 5 successive weeks. Ten days after the final injection, blood was obtained from the auricular vein. The specificity of the antiserum was examined by a double-immunodiffusion method and ELISA. The crossreactivity of anti-chicken CA-III for chicken CA-II, canine CA-I, and canine CA-III was examined by ELISA. Chicken CA-II [19], canine CA-I [20], and canine CA-III [9] had been previously purified. Immunoglobulin $G$ (IgG) was purified using Protein A.

\section{Biotinylation of chicken CA-III}

A 2-mL volume of a solution containing $5 \mathrm{mg} / \mathrm{mL}$ purified chicken CA-III was incubated for $4 \mathrm{~h}$ at $25^{\circ} \mathrm{C}$ with $4.54 \mathrm{mg}$ of biotin (sulfosuccinimidyl N-[N-(D-biotinyl)-6-aminohexanoyl]-6-aminohexanoate; Dojindo Laboratories, Kumamoto, Japan) in $0.08 \mathrm{~mL}$ of $10 \mathrm{~m} M$ HEPES buffer (pH 8.5) (Dojindo Laboratories). Conjugates were then were dialyzed extensively against PBS (pH 7.5).

\section{Blood samples}

Blood samples of clinically normal male WL-chickens (SPF, Line M) at $3(\mathrm{n}=9), 10(\mathrm{n}=9), 12(\mathrm{n}=10), 17(\mathrm{n}=$ 10), $20(\mathrm{n}=10), 27(\mathrm{n}=5), 30(\mathrm{n}=5), 52(\mathrm{n}=5)$, and 59 $(n=5)$ weeks of age were purchased from Nisseiken Co., Ltd. (Tokyo, Japan). Blood samples obtained from clinically normal female WL-chickens (LOHMAN LSL-LITE) at $1(\mathrm{n}=20), 3(\mathrm{n}=20), 9(\mathrm{n}=19), 12(\mathrm{n}=20), 16(\mathrm{n}=$ 20), $21(\mathrm{n}=20), 25(\mathrm{n}=20), 31(\mathrm{n}=20), 49(\mathrm{n}=19), 63$ $(\mathrm{n}=20), 69(\mathrm{n}=20), 73(\mathrm{n}=20), 80(\mathrm{n}=20)$, and $93(\mathrm{n}=$ 20) weeks of age were provided by egg farm (Isogaya Yokeien, Tochigi, Japan). WL-chickens were housed in a conventional curtain side wall cage house with four hens per cage. The forced molt was induced at 64 and finished at 67 weeks of age of all female WL-chickens by the method to reduce quantity and a calorie of the diet. The lighting hours for the force molted group was reduced to 8 hours.

Blood samples were take from individuals of different ages at the same occasion. Blood samples were mixed with lithium heparin and centrifuged at 4,500 $\times$ g for $15 \mathrm{~min}$ at $4^{\circ} \mathrm{C}$ to separate plasma from erythrocytes. Erythrocytes were lysed with an equal volume of distilled water and centrifuged at 27,000 $\times \mathrm{g}$ for $30 \mathrm{~min}$ at $4^{\circ} \mathrm{C}$. Hemolysates and plasma were stored at $-20^{\circ} \mathrm{C}$ until analyzed. Plasma with evidence of hemolysis was not used for CA-III measurements. Samples (0.1 mL volume) of hemolysate at dilutions from 1:4,000 to $1: 16,000$ and plasma at dilutions from 1:5 to 1:10 in buffer A were subjected to ELISA in duplicate.

The egg-laying rates of WL-chicken at the ages of 21 , $25,31,49,63,69,73,80$, and 93 weeks were approximately 20, 94, 94, 95, 91, 2, 63, 91, and $82 \%$, respectively. The egg-laying rate was $0 \%$ at the ages of $1,3,9,12$, and 16 weeks.

\section{Measurement of CA-III levels}

The concentrations of CA-III in chicken hemolysates and plasma were ascertained by the competitive ELISA method. A flat-bottom micro-ELISA plate (Nunc Immuno-plate, Maxisorp, Roskilde, Denmark) was coated for $16 \mathrm{~h}$ at $4^{\circ} \mathrm{C}$ with $0.1 \mathrm{~mL} /$ well of anti-chicken CA-III IgG dissolved in $0.1 M \mathrm{NaHCO}_{3}$ (pH 9.6). Plates were then washed 3 times with $0.3 \mathrm{~mL} /$ well PBS and incubated at $23^{\circ} \mathrm{C}$ for $30 \mathrm{~min}$ with $0.2 \mathrm{~mL} /$ well $0.5 \%$ BSA in $0.05 \mathrm{M}$ Tris- $\mathrm{HCl}$ (pH 8.0) for blocking. Each well was then washed 3 times with $0.3 \mathrm{~mL} /$ well PBS-Tween. The CA-III standards (6-400 ng/mL), biotinylated chicken CA-III, chicken plasma and hemolysates samples were diluted with buffer A, and ELISAs were performed in duplicate. For competition ELISAs, the biotinylated CA-III was mixed with the CA-III standards or the hemolysate and incubated for $16 \mathrm{~h}$ at $4^{\circ} \mathrm{C}$. Each well was then washed 3 times with PBSTween and incubated with $0.1 \mathrm{~mL} /$ well of avidin and biotinylated horseradish peroxidase complex (ABC reagent, Wako Pure Chemical Industries Ltd., Tokyo, Japan) diluted 1:100 with PBS-Tween. After $30 \mathrm{~min}$, each well was washed 3 times with PBS-Tween. Peroxidase activity was measured after addition of $0.1 \mathrm{~mL} /$ well ABTS microwell peroxidase substrate system (Kirkegaard \& Perry Laboratories Inc,). The ABTS substrate system contains 2,2'-azino-di-(3-ethylbenzthiazoline-6-sulfonate) and $\mathrm{H}_{2} \mathrm{O}_{2}$ at a concentration of $0.3 \mathrm{~g} / \mathrm{L}$ and $0.01 \%$ in a glycine/citric acid buffer, respectively. After $10 \mathrm{~min}, 0.1 \mathrm{~mL} /$ well of $1 \%$ SDS was added to terminate the enzyme reaction and the absorbance at $405 \mathrm{~nm}$ was read on an automatic ELISA reader (SH-1000, Corona Electric Co., Ltd., Ibaraki, Japan).

Several experiments were performed to optimize the assay conditions. First, the microplates were coated with several different concentrations of antibody to generate calibration curves. A concentration of $10 \mu \mathrm{g} / \mathrm{mL}$ coating antibody was chosen for the standard assay. The assay precision was evaluated using standard samples of $400 \mathrm{ng} / \mathrm{mL}, 200 \mathrm{ng} / \mathrm{mL}, 100 \mathrm{ng} / \mathrm{mL}, 50 \mathrm{ng} / \mathrm{mL}, 25 \mathrm{ng} /$ $\mathrm{mL}, 12 \mathrm{ng} / \mathrm{mL}$ and $6 \mathrm{ng} / \mathrm{mL}$, each assayed 5 times in 1 assay run.

\section{Protein assay}

The concentrations of aliquots of purified chicken CAIII were determined using the Bio-Rad DC protein assay kit (Bio-Rad Laboratories, Hercules, CA, USA). Bovine 
serum albumin (kit catalog number 500-0111, Bio-Rad Laboratories) was used as a standard.

\section{Hemoglobin assay}

The hemoglobin concentrations in the hemolysates were measured by the sodium lauryl sulfate-hemoglobin method using a hemoglobin B test (Wako Pure Chemical Industries Ltd., Tokyo, Japan).

\section{Statistical analysis}

Values are expressed as means \pm SD. Statistical differences in the CA-III levels of male and female chicken erythrocytes and plasma were analyzed using Student's t-test. Statistical differences in the CA-III levels of each age group were evaluated using a one-way analysis of variance (ANOVA) followed by the Bonferroni post-hoc test. Simple linear regression analysis was used to estimate the relationship between the level of CA-III and the egg-laying percentage. A significance level of $p<0.05$ was used.

\section{Results}

\section{Purification and characterization of chicken CA-III}

Elution profiles were plotted for each step of the purification process. The fourth peak (CM-Fr 1) of the CM-cellulose chromatography column contained CA activity (Figure 1). When fractions of CM-Fr 1 were further purified on a Sephacryl S-200 HR column, the main peak (Fr.

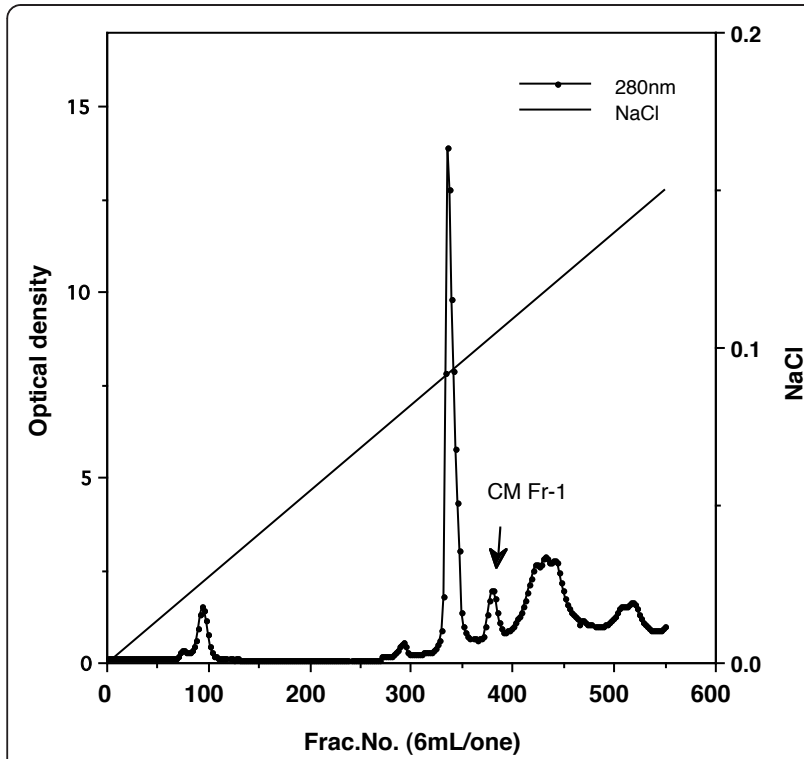

Figure 1 Chromatogram illustrating elution of proteins in muscle tissue extract from a healthy chicken from a carboxymethyl cellulose column $(3.4 \times 30 \mathrm{~cm})$ with a $0 M$ to $0.05 \mathrm{M} \mathrm{NaCl}$ gradient. The total volume of the gradient was 2,500 $\mathrm{mL}$. Optical density of each fraction was determined at $280 \mathrm{~nm}$. The fourth peak (CM-Fr 1) of the CM-cellulose chromatography column contained CA activity.
A) proved to contain chicken CA-III (Figure 2). Figure 3 shows the column electrofocusing elution patterns of chicken CA-III. Purified chicken CA-III produced a $28 \mathrm{kD}$ band by SDS-PAGE analysis (Figure 4). Purified chicken CA-III was analyzed by thin-layer isoelectric focusing (Figure 5) and produced a single band with an isoelectric point of 9.3.

A clear partial amino acid sequence (NYPMA) was obtained from digested chicken CA-III. A FASTA search of the SWISS-PROT databank revealed that this sequence was $100 \%$ identical to the carbonic anhydrase of cyanobacteria; it also showed strong similarity to the $\mathrm{N}$-terminal carbonic anhydrase III of red jungle fowl (accession No. XM_418320; amino acids 23-27: NYPTA). The substitution of methionine $(\mathrm{M})$ for threonine $(\mathrm{T})$ may be attributable to a single nucleotide difference (AG vs. AGㅡ) between leghorn chickens and red jungle fowl.

The specific activity of purified chicken CA-III was 410 units/mg of protein as measured by the method of Wilbur and Anderson [18]. Therefore, we concluded that this isolated protein was carbonic anhydrase III of the White Leghorn chicken.

\section{Specificity of anti-chicken CA-III serum}

Antibodies against chicken CA-III were produced in rabbits. The specificity of the antiserum was evaluated by double immunodiffusion. The antiserum to chicken CA-

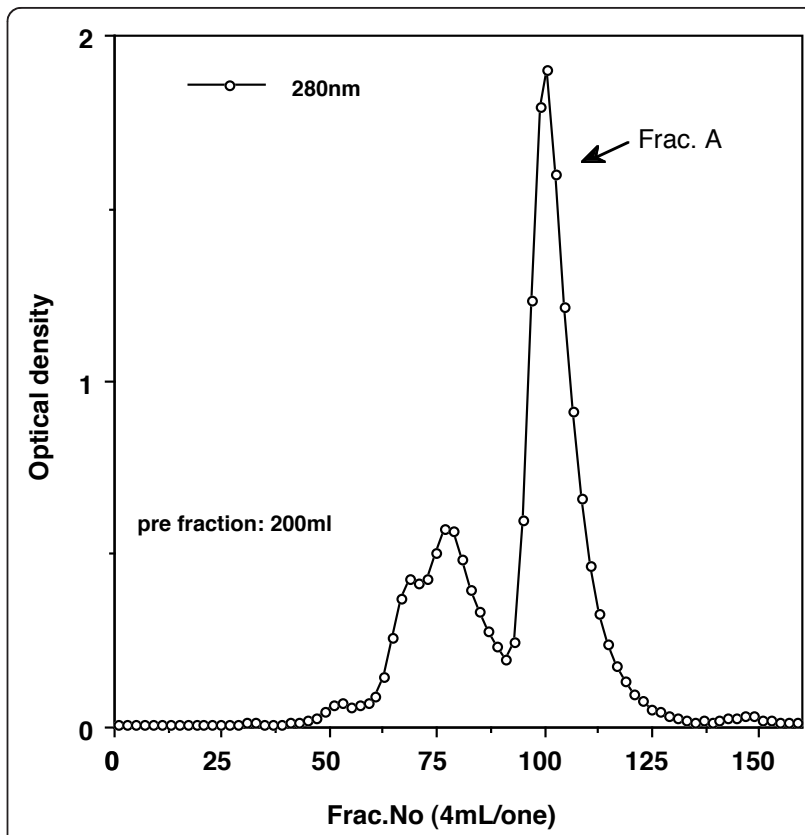

Figure 2 Results of Sephacryl S-200 HR column $(5.0 \times 100 \mathrm{~cm})$ gel filtration of the CM Fr-1 peak in Figure 1. The main peak (Fr. A) proved to contain chicken CA-III. Starting buffer: $0.05 \mathrm{M}$ Tris-HCl ( $\mathrm{pH}$ 8.0) containing $0.5 \mathrm{M} \mathrm{NaCl}$. Absorbance values were read at 280 $\mathrm{nm}$. Flow rate: $20 \mathrm{~mL} / \mathrm{h}$. 

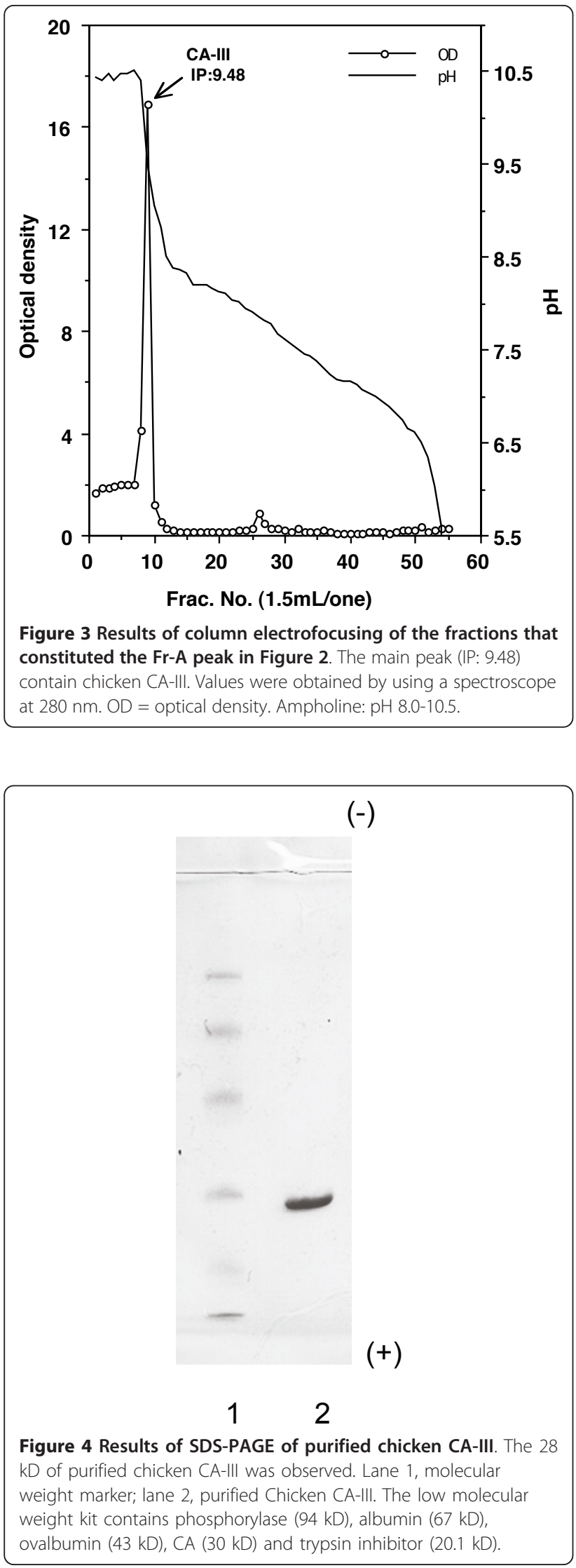

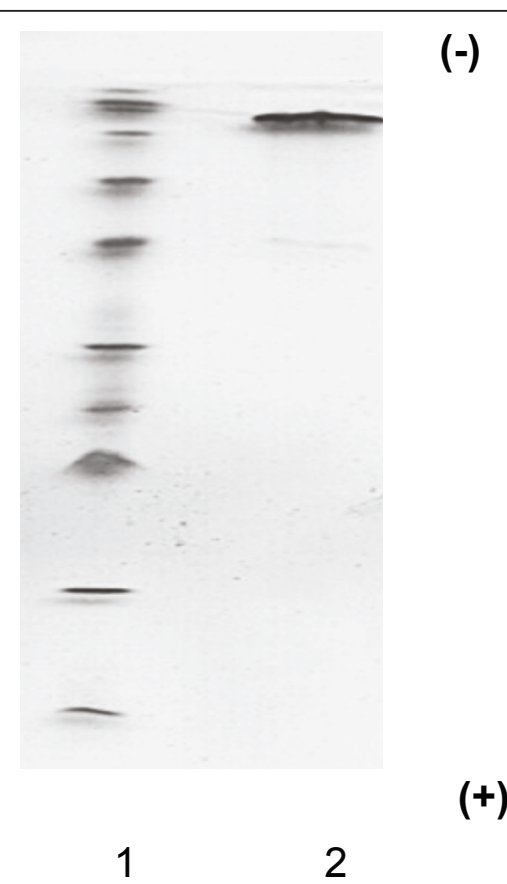

Figure 5 IEF-PAGE of purified chicken CA-III. The single bond of purified chicken CA-III was observed. Lane 1, high pl calibration markers. The high isoelectric point kit contains $\beta$-lactoglobulin A (5.20), bovine CA-B (5.85), human CA-B (6.55), horse myoglobinacidic band (6.85), horse myoglobin-basic band (7.35), lentil lectinacidic band (8.15), lentil lectin-middle band (8.45), lentil lectin-basic band (8.65), and trypsinogen (9.30); lane 2, chicken CA-III.

III formed a single precipitin line with crude chicken muscle extract and purified chicken CA-III, and the precipitin line fused completely (data not shown). Antichicken CA-III does not react with chicken CA-II [19] or canine CA-I, CA-II, or CA-III $[9,20]$. Figure 6 shows the results of western blotting for CA-III on purified chicken CA-III, male WL-chicken hemolysate, and female WL-chicken hemolysate. The molecular weight of these bands was approximately 28,000. Using the ELISA system developed in this study, $40 \mu \mathrm{g} / \mathrm{mL}$ of canine CA-III reacts only slightly with anti chicken CA-III, and the cross reactivity was $0.05 \%$. By contrast, $40 \mu \mathrm{g} / \mathrm{mL}$ each of chicken CA-II, canine CA-I, and canine CA-II, did not react with anti-chicken CA-III. These data indicate that the antibody against chicken CA-III produced in the present study is specific to chicken CA-III.

\section{Measurement of CA-III}

Typical standard curves were plotted for a range of CAIII solutions $(2-400 \mathrm{ng} / \mathrm{mL}$ ) (Figure 7). The coefficients of variation $(\mathrm{CV})$ for these solutions were as follows: $400 \mathrm{ng} / \mathrm{mL}, \mathrm{CV}=4.3 \% ; 200 \mathrm{ng} / \mathrm{mL}, \mathrm{CV}=4.8 \% ; 100 \mathrm{ng} /$ $\mathrm{mL}, \mathrm{CV}=2.4 \% ; 50 \mathrm{ng} / \mathrm{mL}, \mathrm{CV}=6.8 \% ; 25 \mathrm{ng} / \mathrm{mL}, \mathrm{CV}=$ $3.6 \% ; 12 \mathrm{ng} / \mathrm{mL}, \mathrm{CV}=3.1 \% ; 6 \mathrm{ng} / \mathrm{mL}, \mathrm{CV}=2.4 \%$. 
The concentrations of CA-III in hemolysate samples from female WL-chickens (1-93 weeks old) and male WL-chickens (3-59 weeks old) were assayed using competitive ELISA, and the results (mean $\pm \mathrm{SD}$ ) for developmental changes are shown in Figure 8. The levels of CAIII in female chicken erythrocytes ( 1 week old) were $4.6 \pm$ $1.5 \mu \mathrm{g} / \mathrm{g}$ of $\mathrm{Hb}$, and this level did not change until 16 weeks of age. From 21 weeks of age, the CA-III levels in female WL-chickens increased significantly at 25,31 , 49 , and 63 weeks of age $(\mathrm{p}<0.01)$. The CA-III in female chicken erythrocytes approximately doubled between 1 and 21 weeks of age. The level of CA-III in hemolysate from female WL-chickens peaked at 63 weeks of age $(11.8 \pm 3.3 \mu \mathrm{g} / \mathrm{g}$ of $\mathrm{Hb})$. The CA-III then decreased gradually until 73 weeks of age and increased again until 93 weeks of age $(8.6 \pm 2.6 \mu \mathrm{g} / \mathrm{g}$ of $\mathrm{Hb})$. Simple linear regression analysis showed a significant association between the level of CA-III in erythrocytes and the egg-laying rate from 21 to 63 weeks of age in WL-chickens $(\mathrm{p}<0.01)$.

The mean level of CA-III in erythrocytes from 3week-old male WL-chickens was $2.4 \pm 0.8 \mu \mathrm{g} / \mathrm{g}$ of $\mathrm{Hb}$. This level was only half that measured in erythrocytes from female WL-chickens at the same age. The CA-III level in erythrocytes of male WL-chickens did not change significantly from 3 to 27 weeks of age. The mean levels of CA-III in male WL-chicken erythrocytes at 30,52 , and 59 weeks of age were less than that at 20 weeks of age $(\mathrm{p}<0.05)$.

There was a statistically significant difference $(\mathrm{p}<$ 0.01 ) between CA-III levels in erythrocytes from female and male WL-chickens.

The plasma concentrations of CA-III in WL-chickens are shown in Table 1 (male) and Table 2 (female). The plasma concentration of CA-III in male WL-chickens did not change significantly between 3 and 60 weeks of age. The plasma concentration of CA-III in female WLchickens was $60 \mathrm{ng} / \mathrm{mL}$ at 1 weeks of age and increased at 3 weeks of age but did not change significantly between 3 weeks and 80 weeks of age.

Simple linear regression analysis did not show any significant association between the plasma level of CA-III and the egg-laying rate in female WL-chickens from 16 to 63 weeks of age $(p>0.5)$. There was a significant difference $(p<0.01)$ in plasma CA-III levels between female and male WL-chickens.

\section{Discussion}

\section{Identification of chicken CA-III}

In the present study, we developed a relatively simple procedure for purification of CA-III from chicken muscle. The purity of the CA-III was verified by SDS-PAGE and IEF-PAGE analyses. A clear partial amino acid sequence (NYPMA) from digested chicken CA-III was 

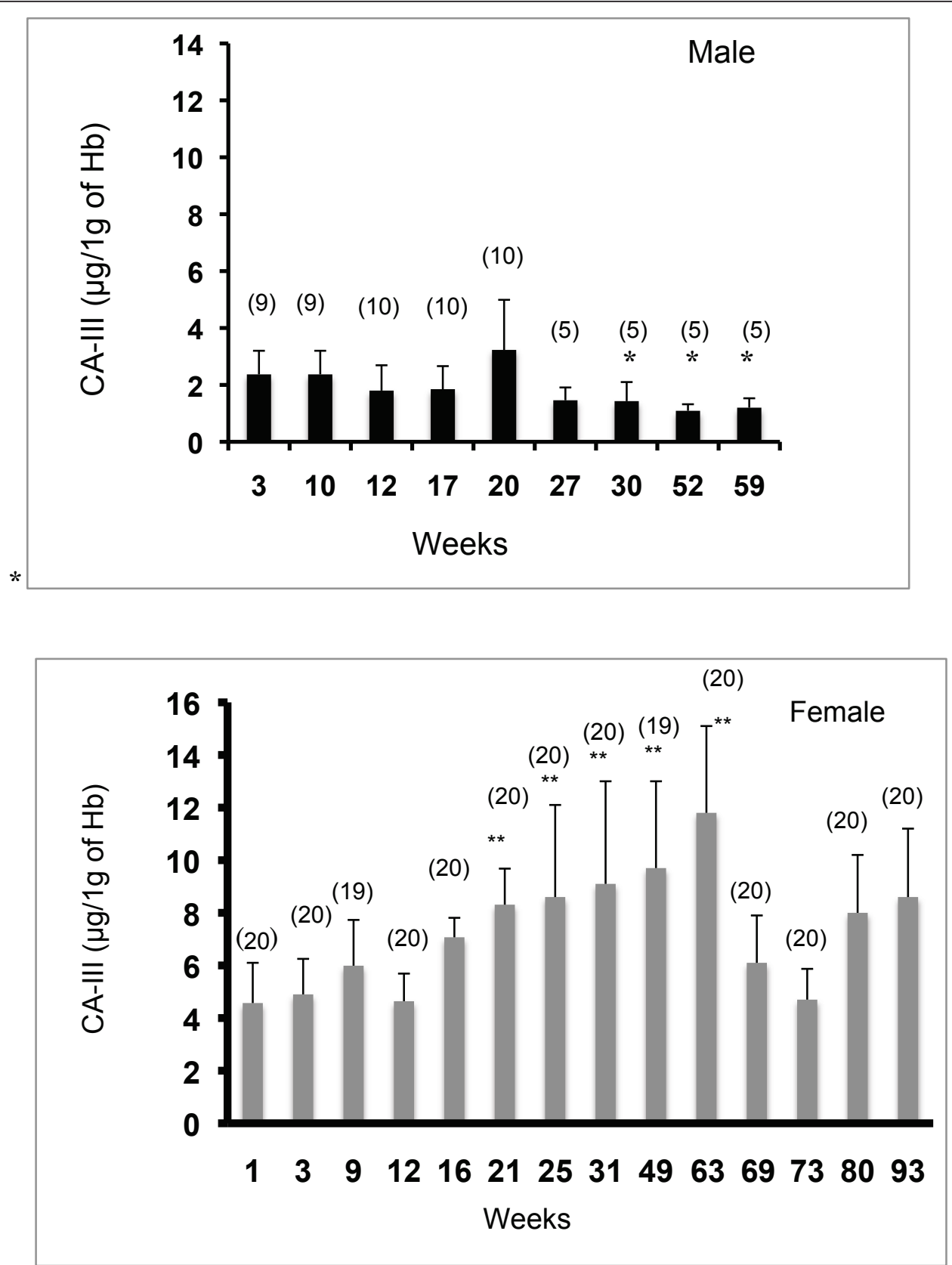

Figure 8 Developmental changes in erythrocyte CA-III levels in male and female WL-chickens. Means and standard deviations of erythrocyte CA-III concentrations ( $\mathrm{\mu g} / \mathrm{g}$ of $\mathrm{Hb}$ ) in male (top) and female (bottom) WL-chickens. The number of samples is shown on the column. The CA-III level in erythrocytes of male WL-chickens did not change significantly from 3 to 27 weeks of age. The mean levels of CA-III in male WL-chicken erythrocytes at 30,52, and 59 weeks of age were less than that at 20 weeks of age $(p<0.05)$. The levels of CA-III in female chicken erythrocytes ( 1 week old) did not change until 16 weeks of age. From 21 weeks of age, the CA-III levels in female WL-chickens increased significantly at 25, 31, 49, and 63 weeks of age. The level of CA-III in hemolysate from female WL-chickens peaked at 63 weeks of age. The CA-III then decreased gradually until 73 weeks of age and increased again until 93 weeks of age. : $: p<0.05, * *: p<0.01$.

$100 \%$ identical to the carbonic anhydrase of cyanobacteria according to a FASTA search of the SWISS-PROT databank. The reported specific activities of equine and canine CA-III are 400 units/mg protein and 370 units/ $\mathrm{mg}$ protein, respectively $[5,9]$. These specific activities are similar to the 410 units/mg protein measured for CA purified from chicken muscle in this study; taken together, these findings are consistent with the conclusion that the low-activity CA isozyme purified from chicken muscle in the present study is CA-III. 
Table 1 The concentration of CA-III in the plasma from male WL-chicken from 3 weeks of age until 59 weeks olds

\begin{tabular}{llc}
\hline Age (week) & $\mathbf{n}$ & $\begin{array}{c}\text { CA-III }(\mathbf{n g} / \mathbf{m L}) \\
\text { (mean } \pm \text { SD) }\end{array}$ \\
\hline 3 & 8 & $58 \pm 17$ \\
10 & 8 & $98 \pm 22$ \\
12 & 7 & $107 \pm 25$ \\
17 & 10 & $105 \pm 37$ \\
20 & 10 & $88 \pm 36$ \\
27 & 4 & $67 \pm 34$ \\
30 & 5 & $70 \pm 52$ \\
52 & 5 & $64 \pm 9.0$ \\
59 & 4 & $88 \pm 24$ \\
\hline
\end{tabular}

The plasma concentration of CA-III in male WL-chickens did not change significantly between 3 and 60 weeks of age.

\section{CA-III levels in erythrocytes}

This is the first description of CA-III levels in chicken erythrocytes. Chicken erythrocytes contain CA-II but not CA-I [21]. In the present study, it is apparent that chicken erythrocytes also contain CA-III. Erythrocytes from female chickens (i,e., 25 weeks old) contained about $1 / 22,000$ as much CA-III as CA-II [19]. The level of CA-III in human erythrocytes was previously reported to be $147 \pm 17 \mu \mathrm{g} / \mathrm{g}$ of $\mathrm{Hb}$ [22]. The level of CA-III in equine erythrocytes was previously reported to be 0.32 $\pm 0.11 \mu \mathrm{g} / \mathrm{g}$ of $\mathrm{Hb}[23]$, and the mean concentration of CA-III in canine erythrocytes was previously reported to

Table 2 The concentration of CA-III in the plasma from female WL-chicken from 1 weeks of age until 93 weeks old

\begin{tabular}{clc}
\hline Age (week) & $\mathbf{n}$ & $\begin{array}{c}\text { CA-III } \\
\text { (ng/mL) } \\
\text { (mean } \pm \text { SD) }\end{array}$ \\
\hline 1 & 16 & $60 \pm 21^{*}$ \\
3 & 15 & $141 \pm 30$ \\
9 & 15 & $166 \pm 30$ \\
12 & 15 & $174 \pm 27$ \\
16 & 13 & $166 \pm 40$ \\
21 & 10 & $141 \pm 58$ \\
25 & 5 & $167 \pm 53$ \\
31 & 5 & $159 \pm 46$ \\
49 & 5 & $161 \pm 39$ \\
63 & 10 & $161 \pm 32$ \\
39 & 16 & $152 \pm 43$ \\
73 & 13 & $146 \pm 36$ \\
80 & 18 & $122 \pm 30$ \\
93 & 7 & $105 \pm 40^{*}$
\end{tabular}

The plasma concentration of CA-III in female WL-chickens was $60 \mathrm{ng} / \mathrm{mL}$ at 1 weeks of age and increased about twofold between 1 and 3 weeks of age. After the initial increase, the plasma CA-III level did not change significantly from 3 to 80 weeks of age.

*: $p<0.01$. be $4.9 \mu \mathrm{g} / \mathrm{g}$ of $\mathrm{Hb}$ [9]. The chicken erythrocyte CA-III level was therefore 29 times higher than the equine level and 2 times higher than the canine level but only 1/16 of the human level.

To confirm whether the level of CA-III in chicken erythrocytes changes during development, erythrocytes were analyzed for CA-III by ELISA. Both developmental changes and sex differences in CA-III levels in chicken erythrocytes were observed. In 21-63-week-old female chickens, erythrocyte CA-III levels were high and correlated with the high level of egg-production efficiency. Tanabe et al. [24] reported that positive correlations were observed between the egg production rate and luteinizing hormone $(\mathrm{LH})$, progesterone, and testosterone concentrations in the chicken plasma.

Hoshino et al.[25] reported that corticosterone, thyroxine (T4), triiodthyronine (T3), and reverse triiodthyronine (rT3) concentrations in the chicken plasma increased during the molt. While, LH, estradiol, and progesterone concentrations declined during the molt, and these declines were coincident with the cessation of egg production. In the present study, the forced molt was induced at 64 weeks and stopped at 67 weeks. At the ages of 63, 69, 73, 80, and 93 weeks, the rates of egg laying were about $91,2,63,91$, and $82 \%$, respectively.

The dog and mature rat exhibit sexual dimorphism in the expression of CA-III in the liver, but the horse, cow, and immature rat do not [17]. In the present study, chicken erythrocyte CA-III was sexually dimorphic. The erythrocyte CA-II level was previously reported to be much higher in female than in male chickens [19]. The erythrocyte CA-III levels in the female chickens decreased significantly from 69 to 73 weeks of age. The rates of egg laying were $2 \%$ at 69 weeks of age and $63 \%$ at 73 weeks of age, respectively. This suggests that the synthesis of CA-III in the erythrocytes at 69 and 73 weeks of age was affected by the forced molt. The synthesis of CA-III within the erythrocyte of laying chicken was probably control by these hormones. However, there was no evidence that these hormones stimulated the synthesis of the CA-III within the erythrocyte of laying chicken. Further investigations of physiological functions of CA-III in the erythrocytes of chicken are needed.

Everaert et al. [26] reported that injection of acetazolamide (ATZ), an inhibitor of CA, increased blood $\mathrm{PCO}_{2}$ and decreased blood $\mathrm{pH}$ in both control and $\mathrm{CO}_{2}$-incubated chicken embryos. ATZ also increased blood bicarbonate concentrations in chicken embryos exposed to normal atmosphere and in day 12 embryos exposed to high $\mathrm{CO}_{2}$. They concluded that no single process (e.g., $\mathrm{CO}_{2}$ buffering by blood CAs) could explain the increase of both $\mathrm{CO}_{2}$ and $\mathrm{HCO}_{3}{ }^{-}$in response to $\mathrm{ATZ}$ and hypothesized the existence of an isozyme other than CA-II in erythrocytes. Although 
CA-III shows low $\mathrm{CO}_{2}$ hydration activity, it has greater resistance to inhibition by ATZ [1]. In the present study, we were the first to find CA-III in chicken erythrocytes.

\section{CA-III levels in plasma}

The human serum CA-III level was previously reported to be $99 \pm 5 \mathrm{ng} / \mathrm{mL}$ [27]. The equine serum CA-III level was previously reported to be $18 \pm 12 \mathrm{ng} / \mathrm{mL}$ [23], and the mean canine serum CA-III level was previously reported to be $17 \pm 10 \mathrm{ng} / \mathrm{mL}(\mathrm{n}=116)$ [9]. In the present study, the plasma CA-III levels in 31-week-old female and 30week-old male chickens were $159 \pm 46 \mathrm{ng} / \mathrm{mL}$ and $70 \pm$ $52 \mathrm{ng} / \mathrm{mL}$, respectively.

Plasma was analyzed by ELISA to determine whether chicken plasma CA-III levels changed during development. Developmental changes and sex differences were observed in chicken plasma CA-III levels. The plasma CAIII level in female chickens increased about twofold between 1 and 3 weeks of age. After the initial increase, the plasma CA-III level did not change significantly from 3 to 80 weeks of age and did not correlate with the egg production efficiency. The plasma CA-III level was also not affected by the forced molt.

By contrast, the plasma CA-III level in 3-week-old male chickens was half that of females at the same age and remained stable throughout development.

\section{Conclusions}

Developmental changes and sex differences were observed in CA-III concentrations in erythrocytes and plasma from WL-chickens. Simple linear regression analysis showed a significant association between the erythrocyte CA-III level and the egg laying rate in 1663-week-old WL-chickens $(\mathrm{p}<0.01)$.

\footnotetext{
Author details

${ }^{1}$ Laboratories of Veterinary Physiology 1, School of Veterinary Medicine, Azabu, University,1-17-71 Fuchinobe, Sagamihara, Kanagawa Japan 252-5201. ${ }^{2}$ Isogaya Yokeien, Kamiishigami, Otawara-shi, Tochigi-ken, Japan 324-0037. ${ }^{3}$ Laboratories of Veterinary Physiology 2, School of Veterinary Medicine, Azabu, University,1-17-71 Fuchinobe, Sagamihara, Kanagawa, Japan 252-5201. ${ }^{4}$ Research Institute of Biosciences, Azabu University, 1-17-71, Fuchinobe, Sagamihara, Kanagawa, Japan 252-5201. ${ }^{5}$ Veterinary Anatomy 2, School of Veterinary Medicine, Azabu University, 1-17-71, Fuchinobe, Sagamihara, Kanagawa, Japan 252-5201.
}

\section{Authors' contributions}

Sample collection design: TN and YT. Sample collection and processing: TN and DY. Survey design and implementation: TN, HO. Survey data entry: TN and $\mathrm{KO}$. Analysis: TN, KO, and KA drafted the paper; the other authors helped to write the paper. All authors read and approved the final manuscript.
}

\section{Competing interests}

The authors declare that they have no competing interests.

Received: 30 April 2011 Accepted: 26 November 2011 Published: 26 November 2011
References

1. Esbaugh AJ, Tufts BL: The structure and function of carbonic anhydrase isozymes in the respiratory system of vertebrates. Respir Physiol Neurobiol 2006, 154:185-198.

2. Fujikawa-Adachi K, Nishimori I, Taguchi T, Onishi S: Human carbonic anhydrase XIV (CA14): cDNA cloning, mRNA expression, and mapping to chromosome 1. Genomics 1999, 61:74-81.

3. Hewett-Emmett D, Tashian RE: Functional diversity, conservation, and convergence in the evolution of the alpha-, beta-, and gamma-carbonic anhydrase gene families. Molecular Phylogenetics and Evolution 1996, 5:50-77.

4. Carter N, Jeffery S, Shiels A, Edwards Y, Tipler T, Hopkinson DA: Characterization of human carbonic anhydrase III from skeletal muscle. Biochem Genet 1979, 17:837-854.

5. Nishita T, Deutsch HF: Isolation of equine muscle carbonic anhydrase in crystalline form. Biochem biophys Res Commun 1981, 103:573-580.

6. Engberg P, Millqvist E, Pohl G, Lindskog S: Purification and some properties of carbonic anhydrase from bovine skeletal muscle. Archs Biochem Biophys 1985, 241:628-638.

7. Holmes RS: Purification, molecular properties and ontogeny of carbonic anhydrase isozymes. Evidence for A, B and C isozymes in avian and mammalian tissues. Eur J Biochem 1977, 78:511-20.

8. Register AM, Koester MK, Noltmann EA: Discovery of carbonic anhydrase in rabbit skeletal muscle and evidence for its identity with " basic muscle protein". J Biol Chem 1978, 253:4143-4152.

9. Nishita T, Matsuura K, Ichihara N, Asari M: Isolation and measurement of carbonic anhydrase isoenzyme III in plasma, sera, and tissues of dogs. Am J Vet Res 2002, 63:229-235.

10. Pullan LM, Noltmann EA: Purification and properties of pig muscle carbonic anhydrase III. Biochim biophys Acta 1985, 839:147-154

11. Räisänen $S R$, Lehenkari $P$, Tasanen $M$, Rahkila $P$, Härkönen $P L$, Väänänen $H K$ : Carbonic anhydrase III protects cells from hydrogen peroxide-induced apoptosis. FASEB J 1999, 13:513-22.

12. Shima K, Tashiro K, Hibi N, Tsukada Y, Hirai H: Carbonic anhydrase III immunohistochemical localization in human skeletal muscle. Acta Neuropath 1983, 59:237-239.

13. Heath R, Jeffery S, Carter ND: Radioimmuoassay of human muscle carbonic anhydrase III in dystrophic states. Clinica Chimica Acta 1982, 119:229-305

14. Hibi N, Shima K, Tashiro K, Tsuzuki K, Tsukada Y, Hirai H: Development of a highly sensitive enzyme-immunoassay for serum carbonic anhydrase III. J Neurol Sci 1984, 65:333-340.

15. Svensson $\mathrm{H}$ : Isoelectric fractionation, analysis, and characterization of ampholytes in natural $\mathrm{pH}$ gradients. I. The differential equation of solute concentrations at a steady state and its solution for simple cases. Acta Chemica Scand 1961, 15:325-341.

16. Cleveland DW: Peptide mapping in one dimension by limited proteolysis of sodium dodecyl sulfate-solubilized proteins. Methods Enzymol 1983, 96:222-229.

17. Nishita T, Matsushita $\mathrm{H}$ : Comparative immunochemical studies of carbonic anhydrase III in horses and other mammalian species. Comp Biochem Physiol B 1988, 91:91-96.

18. Wilbur KM, Anderson NG: Electrometric and colorimetric determination of carbonic anhydrase. J Biol Chem 1948, 176:147-154.

19. Nishita T, Tomita Y, Imanari T, Ichihara N, Orito K, Arishima K: Biochemical and developmental characterization of carbonic anhydrase II from chicken erythrocytes. Acta Vet Scand 2011, 53:16-

20. Nishita $\mathrm{T}$, Kondo $\mathrm{H}$, Ishida $\mathrm{S}$, Ochiai $\mathrm{H}$, Asari M: Isolation and measurement of carbonic anhydrase isoenzymes in erythrocytes of dogs. Am J Vet Res 2000, 61:387-392.

21. Bernstein RS, Schraer R: Purification and properties of an avian carbonic anhydrase from the erythrocytes of Gallus domesticus. J Biol Chem 1972, 247:1306-1322.

22. Heath R, Carter ND, Hewett-Emmett D, et al: Human erythrocytes contain a protein with properties indistinguishiable from skeletal muscle carbonic anhydrase III. Fed Proc 1983, 42:2180.

23. Nishita T, Matsushita H: Carbonic anhydrase III in equine tissues and sera determined by a highly sensitive enzyme-immunoassay. Equine Vet $J$ 1990, 22:247-250.

24. Tanabe $Y$, Nakamura T, Tanase $\mathrm{H}$, Doi O: Comparisons of plasma $\mathbf{L H}$, progesterone, testosterone and estradiol concentrations in male and 
female chickens (Gallus domesticus) from 28 to 1141 days of age. Endocrinol Jpn 1981, 28:605-613.

25. Hoshino S, Suzuki M, Kakegawa T, Imai K, Wakita M, Kobayashi Y, Yamada Y:

Changes in plasma thyroid hormones, luteinizing hormone (LH),

estradiol, progesterone and corticosterone of laying hens during a forced molt. Comp Biochem Physiol A 1988, 90:355-359.

26. Everaert N, Willemsen H, Hulikova A, Brown H, Decuypere E, Swietach P, Bruggeman $\mathrm{V}$ : The importance of carbonic anhydrase II in red blood cells during exposure of chicken embryos to $\mathrm{CO}_{2}$. Respir Physiol Neurobiol 2010, 172:154-161

27. Hoffman EP, Brown RH Jr, Kunkel LM: Dystrophin: the protein product of the Duchenne muscular dystrophy locus. Cell 1987, 51:919-928.

doi:10.1186/1751-0147-53-63

Cite this article as: Nishita et al:: Purification of chicken carbonic anhydrase isozyme-III (CA-III) and its measurement in White Leghorn chickens. Acta Veterinaria Scandinavica 2011 53:63.

\section{Submit your next manuscript to BioMed Central} and take full advantage of:

- Convenient online submission

- Thorough peer review

- No space constraints or color figure charges

- Immediate publication on acceptance

- Inclusion in PubMed, CAS, Scopus and Google Scholar

- Research which is freely available for redistribution

Submit your manuscript at www.biomedcentral.com/submit 